\title{
Switch-When-Safe Multiperiod Mean-Variance Strategies
}

\author{
René Ferland $^{1} \&$ Francois Watier ${ }^{1}$ \\ ${ }^{1}$ Department of Mathematics, Université du Québec à Montréal, Montreal, Canada \\ Correspondence: Francois Watier, Department of Mathematics, Université du Québec à Montréal, P.O. Box 8888, \\ Downtown Station, Montreal, QC., H3C 3P8, Canada. Tel: 1-514-987-3000 ext. 2111. E-mail: \\ watier.francois@uqam.ca
}

Received: January 21, 2013 Accepted: February 27, 2013 Online Published: March 21, 2013

doi:10.5539/ijsp.v2n2p59 URL: http://dx.doi.org/10.5539/ijsp.v2n2p59

This research was supported by a Discovery grant from the Natural Sciences and Engineering Research Council of Canada (NSERC)

\begin{abstract}
In this work, we study the goal-achieving probabilities of a multiperiod mean-variance financial strategy under a switch-when-safe stopping time rule. This stopping time is defined as the first moment, if it occurs, where the investor's cumulative wealth, at this point, can be safely reinvested in a simple bank account in order to meet his financial objective at the end of the investment horizon.
\end{abstract}

Keywords: mean-variance portfolio, multiperiod market model, first-passage time probabilities

\section{Introduction}

Portfolio management theory consists in finding the optimal distribution of wealth among financial assets through a delicate balance between higher returns and lower exposure to risk. In a groundbreaking paper, Markowitz (1952), Nobel prize laureate in economics, proposed the mean-variance portfolio selection model for a myopic investment horizon (single period). Here an investor's objective is to minimize the variance of his terminal wealth under a targeted average return objective. Markowitz's approach is considered by many scholars to be the cornerstone of modern day portfolio theory (see Rubinstein, 2002). The dynamic multiperiod extension of the Markowitz model has been studied extensively since that time, but an explicit expression for the optimal portfolio had not been derived until Li and Ng's (2000) paper.

By design the mean-variance approach works well on average, however it is widely criticized since a real-world investor would experience only one of the many market scenarios. Hence one should also consider the probability of eventually reaching his financial goal. With this in mind, Zhou and Li (2006) devised, in a continuous-time setting, a modified mean-variance portfolio strategy, which we refer to as a switch-when-safe strategy. Basically, an investor follows the usual optimal strategy up to the first (random) moment, if it occurs, where he could reinvest all of his cumulative wealth in a riskless bank account so that it would generate the desired wealth at the end of the investment horizon. They showed that by taking deterministic parameters in a Black Scholes model, which describes stock prices driven by a Brownian motion, gave the following surprising results:

1) the goal-achieving probability is independent of the initial wealth and desired terminal wealth;

2) the goal-achieving probability has an explicit formulation in terms of market parameters and time horizon;

3) the goal-achieving probability has a lower bound of 0.80 .

In this paper, we wish to establish to what extent these properties can be recovered when we consider a more realistic multiperiod (discrete-time) market model where, for example, we are not restricted to assuming a lognormal distribution of stock prices as in a Black-Scholes context.

\section{Switch-When-Safe Portfolios}

\subsection{Multiperiod Market Model and Mean-Variance Strategies}

Consider a financial market with one riskless asset and $n$ risky assets. Let $S_{i}^{0}$ denote the deterministic price of the riskless asset and, for $i=0, \ldots, N$, let $S_{i}^{j}$ be the stochastic price at time $i$ of the $j^{\text {th }}$ risky asset. Let $u_{i}^{j}$ be the amount 
of wealth an investor allocates at the beginning of each time $i=0, \ldots, N-1$ in the $j^{\text {th }}$ risky asset, then assuming that the strategy is self-financing, the wealth process satisfies

$$
x_{i+1}=\left(x_{i}-\sum_{j=1}^{n} u_{i}^{j}\right)\left(\frac{S_{i+1}^{0}}{S_{i}^{0}}\right)+\sum_{j=1}^{n} u_{i}^{j} \frac{S_{i+1}^{j}}{S_{i}^{j}} .
$$

Equivalently by setting $s_{i}=\frac{S_{i+1}^{0}}{S_{i}^{0}}, u_{i}=\left[u_{i}^{1}, \ldots, u_{i}^{n}\right]^{\prime}$ and $P_{i}=\left[\frac{S_{i+1}^{1}}{S_{i}^{1}}-\frac{S_{i+1}^{0}}{S_{i}^{0}}, \ldots, \frac{S_{i+1}^{n}}{S_{i}^{n}}-\frac{S_{i+1}^{0}}{S_{i}^{0}}\right]^{\prime}$

$$
x_{i+1}=s_{i} x_{i}+P_{i}^{\prime} u_{i} \text {. }
$$

A mean-variance strategy $u_{i}$ is the solution to the following stochastic control problem

$$
\min _{u_{i}, 0 \leqslant i \leqslant N-1} \operatorname{VAR}\left(x_{N}\right) \text { s.t. } E\left(x_{N}\right)=z
$$

where $z>x_{0} \prod_{i=0}^{N-1} s_{i}$.

According to $\mathrm{Li}$ and $\mathrm{Ng}(2000)$, under the assumption that $E^{-1}\left(P_{i} P_{i}^{\prime}\right)$ is a positive definite matrix for all time periods, the optimal portfolio is then given by

$$
u_{i}^{M V}=\left[\alpha_{N} \prod_{k=i+1}^{N-1} s_{k}^{-1}-s_{i} x_{i}\right] E^{-1}\left(P_{i} P_{i}^{\prime}\right) E\left(P_{i}\right)
$$

where

$$
\begin{gathered}
\alpha_{N}=x_{0} \prod_{k=0}^{N-1} s_{k}+\frac{z-x_{0} \prod_{k=0}^{N-1} s_{k}}{1-\prod_{k=0}^{N-1}\left(1-B_{k}\right)} \\
B_{k}=E\left(P_{k}^{\prime}\right) E^{-1}\left(P_{k} P_{k}^{\prime}\right) E\left(P_{k}\right) .
\end{gathered}
$$

\subsection{Switch-When-Safe Portfolios and Goal-Achieving Probabilities}

Now consider the following stopping time

$$
\tau_{z}=\inf \left\{0 \leqslant i \leqslant N \mid x_{i} \prod_{k=i}^{N-1} s_{k} \geqslant z\right\}
$$

with the usual conventions $\inf \emptyset=\infty$ and $\prod_{k=j+1}^{j} y_{k}=1$. We define a multiperiod switch-when-safe mean-variance strategy as follows

$$
u_{i}^{S W S}= \begin{cases}u_{i}^{M V}, & \text { if } i<\tau_{z} \wedge N \\ {[0]_{1 \times n},} & \text { else. }\end{cases}
$$

Naturally, we would like to evaluate $P\left(\tau_{z} \leqslant N\right)$, to achieve this we present the following propositions

Proposition 1 A stopped mean-variance wealth reinvested in the riskless asset has a terminal wealth given by

$$
x_{i+1} \prod_{k=i+1}^{N-1} s_{k}=\alpha_{N}-\left(\alpha_{N}-x_{0} \prod_{k=0}^{N-1} s_{k}\right) \prod_{k=0}^{i}\left(1-P_{k}^{\prime} E^{-1}\left(P_{k} P_{k}^{\prime}\right) E\left(P_{k}\right)\right) .
$$

Proof. First, from the wealth Equation (1) we have

$$
x_{i+1} \prod_{k=i+1}^{N-1} s_{k}=x_{i} \prod_{k=i}^{N-1} s_{k}+P_{i}^{\prime} u_{i} \prod_{k=i+1}^{N-1} s_{k} .
$$

By substituting the value of the optimal portfolio (2), setting $Y_{i}=x_{i} \prod_{k=i}^{N-1} s_{k}$ and

$$
C_{i}=P_{i}^{\prime} E^{-1}\left(P_{i} P_{i}^{\prime}\right) E\left(P_{i}\right)
$$

we obtain the following first order linear recursive equation

$$
Y_{i+1}=\left(1-C_{i}\right) Y_{i}+\alpha_{N} C_{i}
$$


which has the solution

$$
Y_{i+1}=Y_{0} \prod_{k=0}^{i}\left(1-C_{k}\right)+\alpha_{N} \sum_{k=0}^{i} \prod_{j=k+1}^{i}\left(1-C_{j}\right) C_{k}
$$

Finally observe that

$$
\sum_{k=0}^{i} \prod_{j=k+1}^{i}\left(1-C_{j}\right) C_{k}=\sum_{k=0}^{i}\left[\prod_{j=k+1}^{i}\left(1-C_{j}\right)-\prod_{j=k}^{i}\left(1-C_{j}\right)\right]=1-\prod_{j=0}^{i}\left(1-C_{j}\right)
$$

thus

$$
Y_{i+1}=\alpha_{N}-\left(\alpha_{N}-Y_{0}\right) \prod_{k=0}^{i}\left(1-C_{k}\right)
$$

Proposition 2 The goal achieving probability of a switch-when safe strategy is given by

$$
P\left(\tau_{z} \leqslant N\right)=P\left(\inf _{0 \leqslant i \leqslant N-1}\left\{\prod_{k=0}^{i}\left(1-C_{k}\right) \leqslant \prod_{k=0}^{N-1}\left(1-B_{k}\right)\right\}\right)
$$

where $B_{k}$ and $C_{k}$ are defined by (3) and (5) respectively.

Proof. From Equation (4), we have

$$
x_{i} \prod_{k=i}^{N-1} s_{k} \geqslant z \Leftrightarrow \prod_{k=0}^{i}\left(1-P_{i}^{\prime} E^{-1}\left(P_{i} P_{i}^{\prime}\right) E\left(P_{i}\right)\right) \leqslant \frac{\alpha_{N}-z}{\alpha_{N}-x_{0} \prod_{k=0}^{N-1} s_{k}}
$$

and therefore

$$
\frac{\alpha_{N}-z}{\alpha_{N}-x_{0} \prod_{k=0}^{N-1} s_{k}}=\frac{x_{0} \prod_{k=0}^{N-1} s_{k}+\frac{z-x_{0} \prod_{k=0}^{N-1} s_{k}}{1-\prod_{k=0}^{N-1}\left(1-B_{k}\right)}-z}{\frac{z-x_{0} \prod_{k=0}^{N-1} s_{k}}{1-\prod_{k=0}^{N-1}\left(1-B_{k}\right)}}=\frac{\frac{1}{1-\prod_{k=0}^{N-1}\left(1-B_{k}\right)}-1}{\frac{1}{1-\prod_{k=0}^{N-1}\left(1-B_{k}\right)}}=\prod_{k=0}^{N-1}\left(1-B_{k}\right)
$$

As in Li and Zhou's continuous-time Black-Scholes model, Equation (6) shows that neither initial wealth or targeted wealth affects the goal-achieving probabilities in a multiperiod setting.

The process appearing in (6) also suggests that in general one might not obtain closed-form formulas for the goalachieving probabilities unless, for example, this process follows a simple dynamic such as a recombinant tree. Nonetheless, in subsection 2.3, we will illustrate that adequate values can be obtained through basic Monte Carlo simulations.

Furthermore a universal $80 \%$ lower bound probability cannot be achieved for all market models and investment horizons. For example, if we consider a single step model $(N=1)$ with a single risky asset, then, from Equation (6), we are reduced to evaluating $P\left(E\left(P_{0}\right) \leqslant P_{0}\right)$. So clearly, if $P_{0}$ follows a symmetrical distribution, then $P\left(E\left(P_{0}\right) \leqslant P_{0}\right)=0.5$. However, in section 3 , we will show that under some assumptions on the market model, we can obtain asymptotical lower bounds (that is for a large number of transaction periods in a given time frame).

\subsection{Numerical Examples}

Example 1 Consider a market model with one risky asset and where the daily log-returns, $\ln \left(\frac{S_{i+1}}{S_{i}}\right)$, are identically distributed and follow a normal distribution with mean $\mu=0.000486$ and standard deviation $\sigma=0.009789$ (Note 1), and furthermore the risky asset has a constant (compounded) daily interest of $r=0.0649 / 365$ (Note 2). Table 1 provides Monte Carlo estimates of the goal-achieving probabilities where the numbers of sample paths are indicated in the first column and the time horizons are indicated in the top row: 
Table 1. Monte Carlo estimates of goal-achieving probabilities for normally distributed log-returns

\begin{tabular}{ccccccc}
\hline$N$ (days) & 5 & 10 & 15 & 20 & 25 & 30 \\
\hline$m=10^{5}$ & 0.7361 & 0.7916 & 0.8083 & 0.8171 & 0.8205 & 0.8210 \\
$m=10^{6}$ & 0.7349 & 0.7897 & 0.8087 & 0.8174 & 0.8209 & 0.8228 \\
$m=10^{7}$ & 0.7356 & 0.7897 & 0.8086 & 0.8171 & 0.8211 & 0.8227 \\
\hline
\end{tabular}

Example 2 In the previous example, the mean and standard deviation characterized the market's dynamics. To better illustrate the effect of the market model's parameters on the hitting time probabilities, we will assume this time that the identically distributed daily $\log$-returns, $\ln \left(\frac{S_{i+1}}{S_{i}}\right)$, follow a four-parameter normal inverse gaussian distribution $N I G(\alpha, \beta, \delta, \mu)$, as seen in Barndorff-Nielsen (1995). We choose mean $\mu=0.000486$, asymmetry parameter $\beta=0$ and generate $m=10^{6}$ sample paths for each time horizons. To easily compare our results, we consider different values for the scale parameter $\delta$ and tail heaviness parameter $\alpha$ all of which generate the same first and second moments $E\left(P_{i}\right)$ and $E\left(P_{i}^{2}\right)$ :

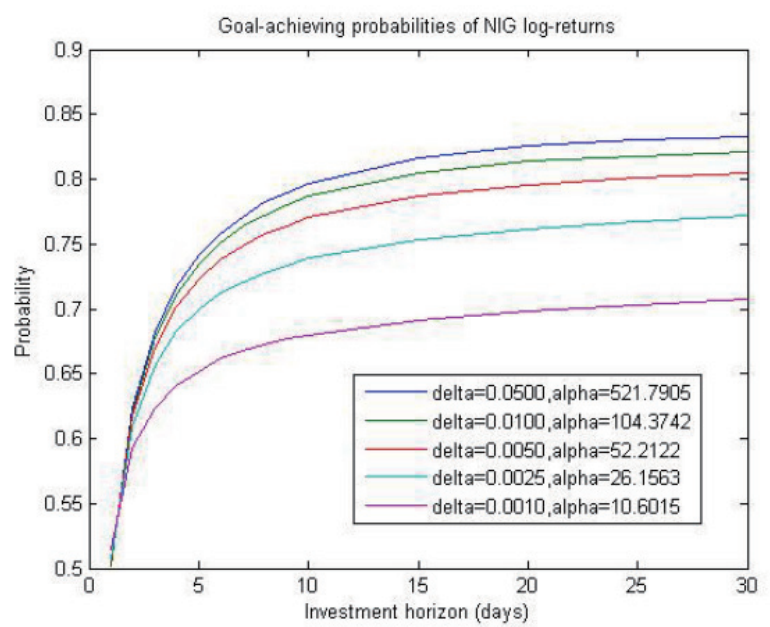

Figure 1. Monte Carlo estimates of goal-achieving probabilities for normal inverse gaussian log-returns

For this type of modeling, Figure 1 suggests that heavier tail distributions (lower values of $\alpha$ ) tend to lead to lower first passage-time probabilities.

Example 3 Finally, to study the effect of mutual dependency in multiple assets models, let us choose an horizon of $N=30$ days and a market model with two risky assets whose joint daily excess rate of return follows a "bivariate binomial tree" process

$$
\left(P_{1, i}, P_{2, i}\right)= \begin{cases}\left(U_{1}, U_{2}\right) & \text { prob. } p_{1} \\ \left(U_{1}, D_{2}\right) & \text { prob. } p_{2} \\ \left(D_{1}, U_{2}\right) & \text { prob. } p_{3} \\ \left(D_{1}, D_{2}\right) & \text { prob. } p_{4}\end{cases}
$$

We consider different values of $p_{1}, p_{2}, p_{3}$, and $p_{4}$ leading to positive, negative or zero correlation between the individual daily excess rate of return of the risky assets and analyze the resulting goal-achieving probabilities.

Let $U_{1}=0.0015, D_{1}=-0.0003, U_{2}=0.0006, D_{2}=-0.0012$, we will consider $p_{1}, p_{2}, p_{3}$, and $p_{4}$ such that the individual excess rate of return of the stocks share the same first and second moment while generating different correlation values. The following table gives Monte Carlo estimates ( $10^{6}$ simulated trajectories) for the goalachieving probabilities:

Table 2. Monte Carlo estimates of goal-achieving probabilities for bivariate tree excess returns

\begin{tabular}{ccccc}
\hline$\left(p_{1}, p_{2}, p_{3}, p_{4}\right)$ & $P\left(\tau_{z} \leqslant N\right)$ & $E\left(P_{j, i}\right)$ & $E\left(P_{j, i}^{2}\right)$ & $\rho\left(P_{1, i}, P_{2, i}\right)$ \\
\hline$\left(\frac{7}{32}, \frac{1}{32}, \frac{17}{32}, \frac{7}{32}\right)$ & 0.8085 & $1.5 \times 10^{-4}$ & $6.3 \times 10^{-7}$ & $\frac{1}{6}$ \\
$\left(\frac{3}{16}, \frac{1}{16}, \frac{9}{16}, \frac{3}{16}\right)$ & 0.8201 & $1.5 \times 10^{-4}$ & $6.3 \times 10^{-7}$ & 0 \\
$\left(\frac{5}{32}, \frac{3}{32}, \frac{19}{32}, \frac{5}{32}\right)$ & 0.8356 & $1.5 \times 10^{-4}$ & $6.3 \times 10^{-7}$ & $-\frac{1}{6}$ \\
\hline
\end{tabular}


In this example, positive (negative) correlation leads to lower (higher) goal-achieving probabilities.

An interesting observation can be made from the second case since it is generated from two independent binomial trees of the form

$$
P_{1, i}=\left\{\begin{array}{cc}
U_{1} & \text { prob. } \frac{1}{4} \\
D_{1} & \text { prob. } \frac{3}{4}
\end{array}, P_{2, i}=\left\{\begin{array}{cc}
U_{2} & \text { prob. } \frac{3}{4} \\
D_{2} & \text { prob. } \frac{1}{4}
\end{array}\right.\right.
$$

If we consider two separate markets with only one of the assets in each, then the individual goal-achieving probabilities would be respectively, for these single asset models, 0.7813 and 0.7886 while in a combined market, where an individual can invest in both assets, the switch-when-safe strategy would produce a higher goal achieving probability of 0.8201 .

\section{Convergence to Continuous-Time Results}

Let $T>0$ and, for each $t \in[0, T]$, set $i=[N t]$, we will consider a financial market consisting of one riskless asset and one risky asset and suppose that the excess rate of return $\left\{P_{i}, i=0, \ldots, N-1\right\}$ are independent and identically distributed.

Proposition 3 Let $m_{N}=E\left[\ln \left(1-C_{i}\right)\right]$ and $s_{N}^{2}=V A R\left[\ln \left(1-C_{i}\right)\right]$, if there exists $\alpha \in R, \beta>0$ and $\gamma<0$ such that for each $i=0, \ldots, N-1$

(Al) $\lim _{N \rightarrow \infty} i m_{N}=\alpha t$;

(A2) $\lim _{N \rightarrow \infty} N s_{N}^{2}=\beta^{2} T$;

(A3) $\lim _{N \rightarrow \infty} N \ln \left(1-B_{i}\right)=\gamma T$;

then

$$
\lim _{N \rightarrow \infty} P\left(\tau_{z} \leqslant N\right)=\Phi\left(\left(\frac{\gamma-\alpha}{\beta}\right) \sqrt{T}\right)+e^{2 \frac{\alpha \gamma}{\beta^{2}} T} \Phi\left(\left(\frac{\alpha+\gamma}{\beta}\right) \sqrt{T}\right)
$$

where $\Phi$ is the cumulative density function of a standardized normal distribution.

Proof. From (6), we have

$$
P\left(\tau_{z} \leqslant N\right)=P\left(\inf _{0 \leqslant i \leqslant N-1}\left\{\sum_{k=0}^{i} \ln \left(1-C_{k}\right) \leqslant N \ln \left(1-B_{k}\right)\right\}\right) .
$$

Now, consider the process

$$
\sum_{k=0}^{i} \ln \left(1-C_{k}\right)-N \ln \left(1-B_{k}\right)=\left(\frac{\sqrt{N} s_{N}}{\sqrt{T}}\right) \frac{\sqrt{T}}{\sqrt{N}} \sum_{k=0}^{i}\left[\frac{\ln \left(1-C_{k}\right)-m_{N}}{s_{N}}\right]+i m_{N}-N \ln \left(1-B_{k}\right)
$$

where $S_{i}=\sum_{k=0}^{i} X_{k}, X_{k}=\frac{\ln \left(1-C_{k}\right)-m_{N}}{s_{N}}$ and consider the partial sums process (linear interpolation)

$$
\left\{\xi_{N}(t)=S_{i}+(N t-i) X_{i+1}, t \in[0, T]\right\} .
$$

From Donsker's invariance principle (see Karatzas \& Shreve, 1991), $\frac{\sqrt{T}}{\sqrt{N}} \xi_{N}$ converges weakly (in distribution) to Brownian motion $W(t)$ on $[0, T]$. Therefore, using assumptions $(A 1),(A 2)$ and $(A 3)$ then

$$
\left(\frac{\sqrt{N} s_{N}}{\sqrt{T}}\right) \frac{\sqrt{T}}{\sqrt{N}} \xi_{N}+i m_{N}-N \ln \left(1-B_{k}\right) \stackrel{\mathcal{L}}{\longrightarrow} \beta W(t)+\alpha t-\gamma
$$

on $[0, T]$. Since the first passage time functional is continuous almost everywhere with respect to the Brownian motion (with drift), using the continuous mapping theorem (see Whitt, 2002) and results on first passage-time of Brownian motion through time-varying boundaries (see Di Nardo et al., 2001), we have

$$
\lim _{N \rightarrow \infty} P\left(\tau_{z} \leqslant N\right)=\Phi\left(\left(\frac{\gamma-\alpha}{\beta}\right) \sqrt{T}\right)+e^{2 \frac{\alpha \gamma}{\beta^{2}} T} \Phi\left(\left(\frac{\alpha+\gamma}{\beta}\right) \sqrt{T}\right) .
$$


Proposition 4 Let $\alpha, \beta$ and $\gamma$ as defined in Proposition 3, if $\alpha<0$ and $\gamma>\alpha$ then for every $T>0$ we have

$$
\lim _{N \rightarrow \infty} P\left(\tau_{z} \leqslant N\right) \geqslant \Phi\left(\sqrt{\frac{\alpha-\gamma}{\alpha+\gamma}}\right)-\frac{\sqrt{\alpha^{2}-\gamma^{2}}}{2 \alpha} \varphi\left(\sqrt{\frac{\alpha-\gamma}{\alpha+\gamma}}\right),
$$

where $\varphi$ and $\Phi$ are respectively the probability and cumulative density functions of a standardized normal distribution.

Proof. Let

$$
f(x)=\Phi\left(\left(\frac{\gamma-\alpha}{\beta}\right) x\right)+e^{2 \frac{\alpha \gamma}{\beta^{2}} x^{2}} \Phi\left(\left(\frac{\alpha+\gamma}{\beta}\right) x\right),
$$

a smooth function defined on $[0, \infty)$ then, using the fact that $\varphi\left(\left(\frac{\gamma-\alpha}{\beta}\right) x\right)=e^{2 \frac{\alpha \gamma}{\beta^{2}} x^{2}} \varphi\left(\left(\frac{\alpha+\gamma}{\beta}\right) x\right)$, we have

$$
\frac{\partial f(x)}{\partial x}=\frac{2 \gamma}{\beta} e^{2 \frac{\alpha \gamma}{\beta^{2}} x^{2}}\left[\varphi\left(\left(\frac{\alpha+\gamma}{\beta}\right) x\right)+\frac{2 \alpha}{\beta} x \Phi\left(\left(\frac{\alpha+\gamma}{\beta}\right) x\right)\right] .
$$

Since $\left.\frac{\partial f(x)}{\partial x}\right|_{x=0}=\frac{2 \gamma}{\beta} \varphi(0)<0, f(0)=1$ and $\lim _{x \rightarrow \infty} f(x)=1$ then $f$ admits at least one local minima. We will show that this minima is unique.

First observe that

$$
\frac{\partial f(x)}{\partial x}=\frac{2 \gamma}{\beta} x e^{2 \frac{\alpha \gamma}{\beta^{2}} x^{2}} g(x),
$$

where

$$
g(x)=\frac{\varphi\left(\left(\frac{\alpha+\gamma}{\beta}\right) x\right)}{x}+\frac{2 \alpha}{\beta} \Phi\left(\left(\frac{\alpha+\gamma}{\beta}\right) x\right) .
$$

Now

$$
\frac{\partial g(x)}{\partial x}=\varphi\left(\left(\frac{\alpha+\gamma}{\beta}\right) x\right)\left[\frac{\alpha^{2}-\gamma^{2}}{\beta^{2}}-\frac{1}{x^{2}}\right]
$$

therefore $g$ is decreasing on $\left[0, \frac{\beta}{\sqrt{\alpha^{2}-\gamma^{2}}}\right]$ and increasing on $\left[\frac{\beta}{\sqrt{\alpha^{2}-\gamma^{2}}}, \infty\right]$, since $\lim _{x \rightarrow 0^{+}} g(x)=\infty$ and $\lim _{x \rightarrow \infty} g(x)=$ 0 there is a unique value $\hat{x} \in\left[0, \frac{\beta}{\sqrt{\alpha^{2}-\gamma^{2}}}\right]$ such that $\left.\frac{\partial f(x)}{\partial x}\right|_{x=\hat{x}}=g(\hat{x})=0$.

Thus, $\hat{x}$ is the global point of minimum of $f$, that is for all $x \in[0, \infty]$,

$$
\begin{aligned}
f(x) & \geqslant f(\hat{x}) \\
& =\Phi\left(\left(\frac{\gamma-\alpha}{\beta}\right) \hat{x}\right)+e^{2 \frac{\alpha \gamma}{\beta^{2}} \hat{x}^{2}} \Phi\left(\left(\frac{\alpha+\gamma}{\beta}\right) \hat{x}\right) \\
& =\Phi\left(\left(\frac{\gamma-\alpha}{\beta}\right) \hat{x}\right)-\frac{\beta}{2 \alpha} e^{2 \frac{\alpha \gamma}{\beta^{2}} \hat{x}^{2}} \frac{\varphi\left(\left(\frac{\alpha+\gamma}{\beta}\right) \hat{x}\right)}{\hat{x}} \\
& =\Phi\left(\left(\frac{\gamma-\alpha}{\beta}\right) \hat{x}\right)-\frac{\beta}{2 \alpha} \frac{\varphi\left(\left(\frac{\gamma-\alpha}{\beta}\right) \hat{x}\right)}{\hat{x}} .
\end{aligned}
$$

Finally, consider

$$
h(y)=\Phi\left(\left(\frac{\gamma-\alpha}{\beta}\right) y\right)-\frac{\beta}{2 \alpha} \frac{\varphi\left(\left(\frac{\gamma-\alpha}{\beta}\right) y\right)}{y}
$$

defined on $\left[0, \frac{\beta}{\sqrt{\alpha^{2}-\gamma^{2}}}\right]$, then

$$
\frac{\partial h(y)}{\partial y}=\frac{\beta}{2 \alpha} \varphi\left(\left(\frac{\gamma-\alpha}{\beta}\right) y\right)\left[\frac{1}{y^{2}}-\frac{\alpha^{2}-\gamma^{2}}{\beta^{2}}\right],
$$


thus $h$ is decreasing on $\left[0, \frac{\beta}{\sqrt{\alpha^{2}-\gamma^{2}}}\right]$ then

$$
\begin{aligned}
f(x) & \geqslant f(\hat{x}) \\
& \geqslant h\left(\frac{\beta}{\sqrt{\alpha^{2}-\gamma^{2}}}\right) \\
& =\Phi\left(\sqrt{\frac{\alpha-\gamma}{\alpha+\gamma}}\right)-\frac{\sqrt{\alpha^{2}-\gamma^{2}}}{2 \alpha} \varphi\left(\sqrt{\frac{\alpha-\gamma}{\alpha+\gamma}}\right) .
\end{aligned}
$$

Example 4 Consider the Cox-Ross-Rubinstein binomial tree model (see Shreve, 2005), a discrete version of the continuous-time Black-Scholes market model. This is a three-parameter financial market model, with $\mu>r>0$ and $\sigma>0$, where the riskless asset's dynamics are given by

$$
\frac{S_{i+1}^{0}}{S_{i}^{0}}=e^{r T / N}
$$

while those of the risky asset's are given by

$$
\frac{S_{i+1}^{1}}{S_{i}^{1}}= \begin{cases}e^{\sigma \sqrt{T / N}} & \text { prob. } p=\frac{e^{\mu T / N}-e^{-\sigma \sqrt{T / N}}}{e^{\sigma \sqrt{T / N}}-e^{-\sigma \sqrt{T / N}}} \\ e^{-\sigma \sqrt{T / N}} & \text { prob. } 1-p\end{cases}
$$

In this case, $\lim _{N \rightarrow \infty} i m_{N}=-\frac{3}{2} \theta^{2} t, \lim _{N \rightarrow \infty} N s_{N}^{2}=\theta^{2} T$ and $\lim _{N \rightarrow \infty} N \ln \left(1-B_{k}\right)=-\theta^{2} T$ where $\theta=\frac{\mu-r}{\sigma}>0$ therefore from (7) we have

$$
\lim _{N \longrightarrow \infty} P\left(\tau_{z} \leqslant N\right)=\Phi\left(\frac{1}{2} \theta \sqrt{T}\right)+e^{3 \theta^{2} T} \Phi\left(-\frac{5}{2} \theta \sqrt{T}\right)
$$

and from (8),

$$
\lim _{N \rightarrow \infty} P\left(\tau_{z} \leqslant N\right) \geqslant \Phi\left(\frac{1}{\sqrt{5}}\right)+\frac{\sqrt{5}}{6} \varphi\left(\frac{1}{\sqrt{5}}\right)>0.80
$$

which is precisely Zhou's result in the continuous-time setting with constant market parameters.

\section{Conclusion}

In this paper, we established, for a general discrete-time market model, fundamental properties of a multiperiod switch-when-safe mean-variance strategy. This is a financial strategy in which an investor follows the optimal mean-variance strategy up to the first moment, if it occurs, at which time the cumulative wealth can be transferred in a simple bank account in order to safely attain the financial objective at the end of the investment horizon. Under the assumption that the excess rates of return are time-independent, surprisingly, the goal-achieving probabilities are not affected by the initial wealth nor targeted wealth. Goal-achieving probabilities are easily obtained through standard Monte Carlo simulations of the first passage time through a fixed boundary of products of time independent random variables. Furthermore, under mild assumptions on the market model, the continuous-time limit of the goal-achieving probability expression converges to an explicit tractable formula involving the cumulative density function of a standardized normal distribution. Finally, in several cases, the continuous-time limit of the goal-achieving probability admits a lower bound.

Further investigation might include a double-barrier problem in which the investor would also introduced a lower threshold for his cumulative wealth when pursuing the mean-variance optimal strategy. A common situation would be a no bankruptcy condition, therefore we could study the probability of first reaching a safe reinvestment level of wealth before hitting possible bankruptcy.

An equally interesting problem would be the study of the goal-achieving probabilities in the case of a multiperiod setting where the excess rate of returns are allowed to be time-dependent (see Schweizer, 1995; Vaillancourt \& Watier, 2005) this would include usual models such as Markov chains and time series. A particularly challenging question with the time-dependency feature would be to know if continuous-time limit results can still be obtained since, in this case, we cannot directly apply Donsker's invariance principle (functional central limit theorem). 


\section{Acknowledgements}

The authors would like to thank the editor, anonymous referees and professor Juli Atherton for their helpful comments and suggestions.

\section{References}

Barndorff-Nielsen, O. (1995). Normal-inverse gaussian processes and the modelling of stock returns. Technical research report No. 300, Department of Theoretical Statistics, University of Aarhus.

Di Nardo, E., Nobile, A. G., Pirozzi, E., \& Ricciardi, L. M. (2001). A computational approach to first-passage-time problems for GaussMarkov processes. Advances in Applied Probability, 33(2), 453-482. http://dx.doi.org/10.1239/aap/999188324

Fergusson, K., \& Platen, E. (2006). Characterization of daily log-returns of a world stock index. Applied Mathematical Finance, 13(1), 19-38. http://dx.doi.org/10.1080/13504860500394052

Karatzas, I., \& Shreve, S. (1991). Brownian motion and stochastic calculus (2nd ed). New York, NY: SpringerVerlag.

Li, D., \& Ng, W. L. (2000). Optimal dynamic portfolio selection: Multiperiod mean-variance formulation. Mathematical Finance, 10(3), 387-406. http://dx.doi.org/10.1111/1467-9965.00100

Li, X., \& Zhou, X. Y. (2006). Continuous-time mean-variance efficiency: The 80\% rule. Annals of Applied Probability, 16(4), 1752-1763. http://dx.doi.org/10.1214/105051606000000349

Markowitz, H. (1952). Portfolio selection. Journal of Finance, 7, 77-91.

Rubinstein, M. (2002). Markowitz's Portfolio selection: A fifty-year retrospective. Journal of Finance, 17(3), 1041-1045. http://dx.doi.org/10.1111/1540-6261.00453

Schweizer, M. (1995). Variance-Optimal Hedging in Discrete Time. Mathematics of Operations Research, 20(1), 1-32. http://dx.doi.org/10.1287/moor.20.1.1

Shreve, S. (2005). Stochastic calculus for finance I: The binomial asset pricing model (1st ed.). New York, NY: Springer-Verlag.

Vaillancourt, J., \& Watier, F. (2005). On an optimal multivariate multiperiod mean-variance portfolio. Mathematical Reports of the Academy of Science. The Royal Society of Canada, 27(3), 92-96.

Whitt, W. (2002). Stochastic-Process Limits. New York, NY: Springer-Verlag.

\section{Notes}

Note 1. The mean and standard deviation values are taken from Fergusson and Platen's (2006) WSI (World Stock Index) for the period from 1970 until 2004.

Note 2. This corresponds to the average Federal funds effective rate for the period from 1970 until 2004 converted to a daily rate. 